\title{
UWB Out-of-Phase Network feeding a 2-Element Impulse Radiating Array
}

\author{
Elena Pancera*, Grzegorz Adamiuk, Thomas Zwick and Werner Wiesbeck \\ Institut für Höchstfrequenztechnik und Elektronik (IHE) \\ Universität Karlsruhe (TH), \\ Kaiserstrasse 12, 76128 Karlsruhe, Germany \\ E-mail: elena.pancera@ihe.uka.de
}

\section{Introduction}

Since 2002, when the FCC has regulated the usage of the frequency spectrum for UWB communications, various antennas and arrays with UWB characteristic have been presented. However, in the majority of cases, arrays are fed by an inphase feeding network and few works deal with Out-of-Phase fed UWB arrays.

The main advantage of having an Out-of Phase fed array is the possibility of having lower level of cross polarization and coupling between the array elements.

In Fig.1, a conceptual block scheme of an Out-of-Phase feeding network is presented. In such a network, in the UWB case, the main problem consists in the realization of the $180^{\circ}$ phase shifter. The most common techniques for its realization deal mainly with narrow band devices, where the $180^{\circ}$ phase shifters are usually realized by lines of $\lambda / 2$ at the center frequency. In the UWB case, this technique is not possible to be applied, due to the large used frequency range. Very few examples of wideband phase shifters are reported [1].

In this paper, an analysis of an Out-of-Phase feeding network, which is suitable to be used in the FCC UWB frequency range, will be presented. Firstly, a study of the parameters that determine the UWB behavior will be performed. Secondly, this feeding network will be applied to an antenna array in order to validate the achievement of low level of cross polarization and low coupling between the array elements. The complete system composed by the array and the feeding network has also been measured through a full 3D characterization and the main results will be here presented. Furthermore, a time domain analysis has also been conducted, in order to evaluate the performance and the influence of the proposed feeding network with respect to the radiated pulses.

\section{Out-of-Phase Feeding Network}

The proposed structure is composed of a power divider with one input line and two output lines. In one of these output lines, a $180^{\circ}$ phase shifter is positioned, in order that the currents at the end of the two lines have $180^{\circ}$ phase difference.

The $180^{\circ}$ phase shifter is composed by a microstrip back to back balun. A first example of microstrip back to back balun can be found in [2].

The presented model is composed by the cascade of two tapered sections, as illustrated in Fig.2, where also the parameters that influence the UWB behaviour are depicted. The cascade of the two tapered sections permits to obtain the UWB behaviour, once the dimensions are correctly set.

A parametrical study has been conducted, in order to evaluate the influence of the different parameters. It has been seen that, once the phase shifter length $\left(x_{1}+x_{2}\right)$ is set, both the parameters $y_{1}$ and $y_{2}$ influence the UWB behaviour of the device. 


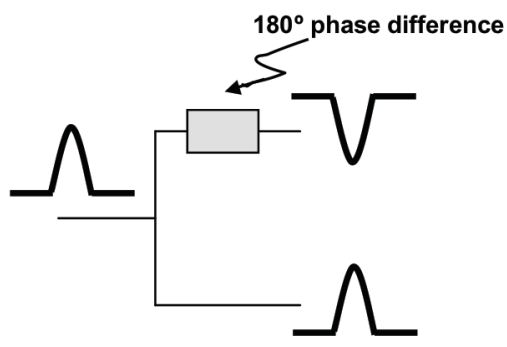

Fig.1: Conceptual block scheme of an Outof-Phase Feeding Network

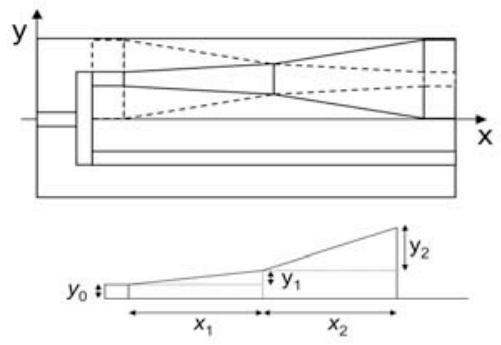

Fig.2: The realized Out-of-Phase feeding network and parametric dimensions.

In Fig. 3 the obtained phase difference between the two output ports is depicted. It is compared to the usage of a classical delay line, whose length is set to be $\lambda / 2$ for $f=6.5 \mathrm{GHz}$, the centre frequency of the UWB interval. As it can be seen, for the proposed structure the difference from the two output lines is $180 \pm 5$ degrees in the whole UWB band, and its behaviour is better than the classical delay line.

A prototype of this feeding network has been realized on a Rogers substrate RO3206 with $\varepsilon_{\mathrm{r}}=6.15$ and thickness $0.64 \mathrm{~mm}$. It has been applied to a twoelement array, which is a modified version of the coplanar monopole [3]. It has been realized on a Rogers substrate with $\varepsilon_{\mathrm{r}}=2.22$ and thickness $1.575 \mathrm{~mm}$ (see Fig.4).

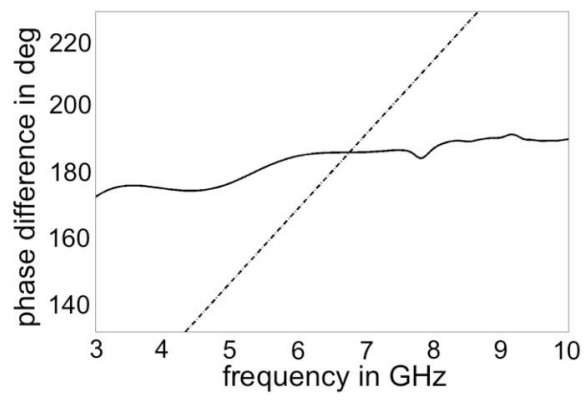

Fig.3:Phase difference of the feeding network (solid line) vs. classical $\lambda / 2$ delay line.

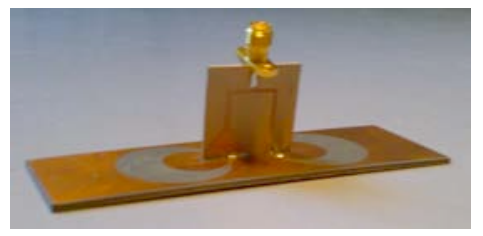

Fig.4: The realized Out-of-Phase feeding Network and the two- element array.

\section{Frequency Domain Analysis}

In order to evaluate the influence of the feeding network together with the antenna, a measurement in an anechoic chamber has been performed with the Device Under Test (DUT) being the system composed by the antenna and the Out-of-Phase feeding network. Letting $U_{\mathrm{tx}}$ be the transmitted voltage and $U_{\mathrm{rx}}$ the received voltage, the transmission coefficient $S_{21}$ between the DUT and a reference antenna is given by

$$
S_{21}(\omega)=\frac{U_{\mathrm{rx}}(\omega)}{U_{\mathrm{tx}}(\omega)}=H_{\mathrm{ref}}(\omega) H_{\mathrm{DUT}}(\omega) \frac{j \omega}{2 \pi r c} e^{-j \omega r / c}
$$

where $H_{\text {ref }}$ and $H_{\text {DUT }}$ are the transfer functions (in terms of effective antenna height) of the reference antenna and of the DUT, respectively, and $r$ is the distance between the two antennas. Hence, the gain of the DUT is

$$
G_{D U T}(\omega)=\frac{\omega^{2}}{\pi c^{2}}\left|H_{\text {DUT }}(\omega)\right|^{2}=4 \pi r^{2}\left|\frac{S_{21}(\omega)}{H_{\text {ref }}(\omega)}\right|^{2}
$$


In figure 5, the measured gain pattern for $\psi=90^{\circ}$ and different angular directions $\theta$ is illustrated for the co-polarization (left) and the cross-polarization component (right). The system presents a very low level of cross-polarization.
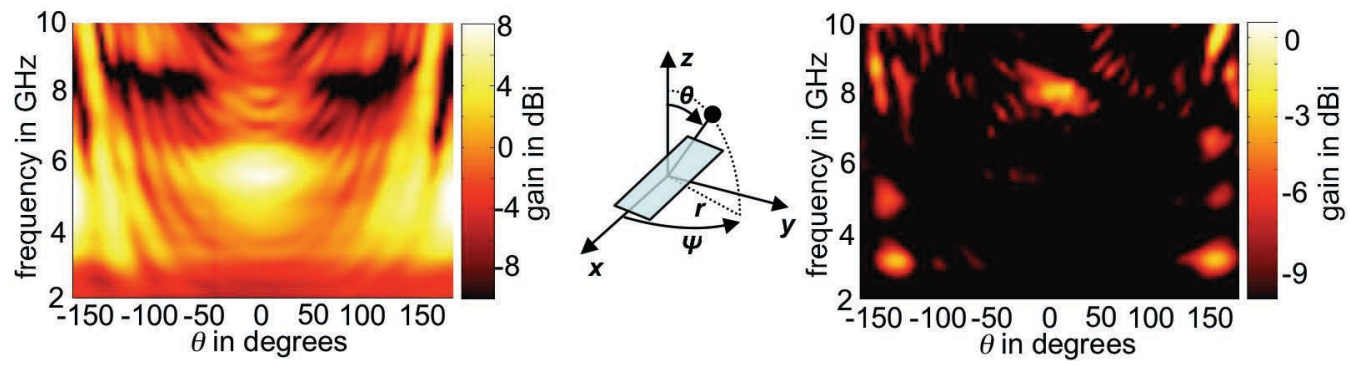

Fig.5: Measured gain pattern for $\psi=90^{\circ}$ and different $\theta$ for the co-polarization (left) and crosspolarization (right) component and the DUT board position with respect to the axes system.

\section{Time Domain Analysis}

The time domain transient response of the DUT can be derived starting from the analytic transfer function in the time domain, defined as [4]

$$
H^{+}(\omega)= \begin{cases}2 H_{\mathrm{DUT}}(\omega) & \omega>0 \\ 0 & \omega \leq 0\end{cases}
$$

The transient response of the DUT is the real part of the inverse Fourier Transform of $H^{+}(\omega)$.

$$
h_{\mathrm{DUT}}(t)=\operatorname{Re}\left[h^{+}(t)\right]
$$

Since $h_{\mathrm{DUT}}$ depends on both the angular directions $\theta, \psi$, as $H_{\mathrm{DUT}}$, in order to evaluate this angular direction dependence, a full 3D measurement of the DUT has been performed. In Fig.6, the obtained results are illustrated. Here, each image represents the absolute value of the vector sum of both the linear polarization components of the envelope of the measured transient response at a particular time. This novel visualization method permits to evaluate the DUT transient response in all the angular directions vs time. As it can be seen, a peak of the transient response appears at approximately $0.8 \mathrm{~ns}$ simultaneously in all angular directions, showing the omni-directionality of the DUT. Its duration is about $0.3 \mathrm{~ns}$, since at $1.2 \mathrm{~ns}$ it has already drastically weakened. After $1.2 \mathrm{~ns}$, it is possible to observe some ringing, whose entity and time duration is very small.

Furthermore, in the Fig.7, the absolute value of the measured transient response for $\psi=90^{\circ}$ and different angular directions $\theta$ is depicted vs. time. The peak duration is very small and it presents low level and duration of the ringing. From both these analysis, the measurements indicates that the realized feeding network does not distort the pulse shape. This is an important result since it proves that this realized network, which has a good frequency domain behavior with an almost constant phase difference of 180 degree, has also a good time domain behavior.

\section{Conclusions}

In this paper, an Out-of-Phase feeding network, which is suitable to be used in the UWB frequency range, has been presented. This feeding network has been used to 


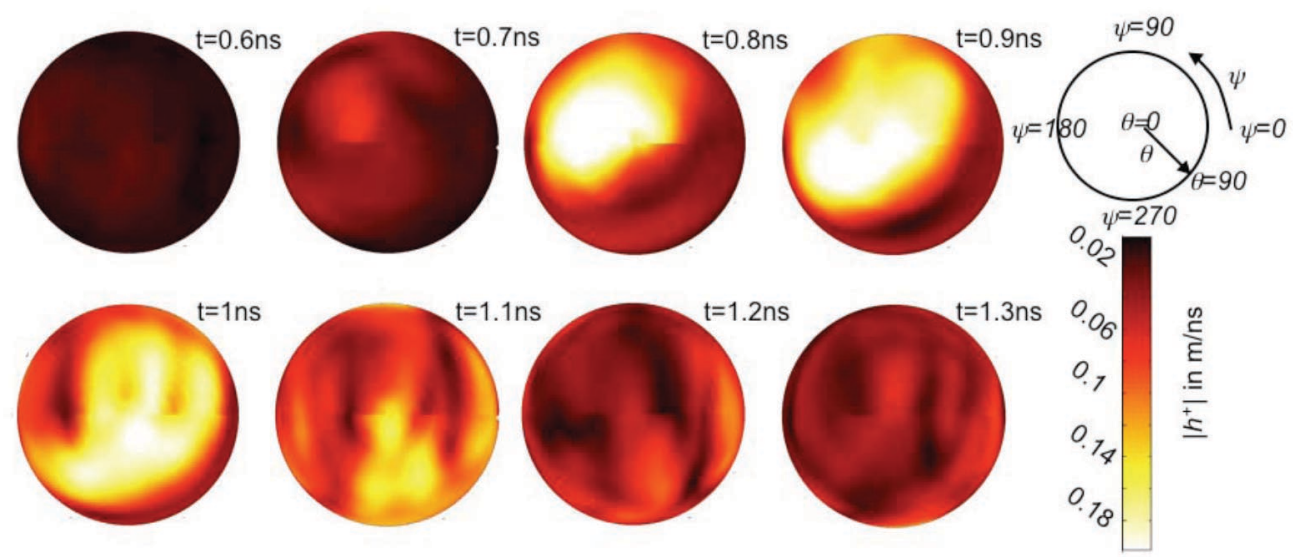

Fig.6: Measured transient response of the DUT for $\psi=-180 \ldots 180 \mathrm{deg}$. and $\theta=0 \ldots 90 \mathrm{deg}$, distance between reference antenna and DUT $10 \mathrm{~m}$.

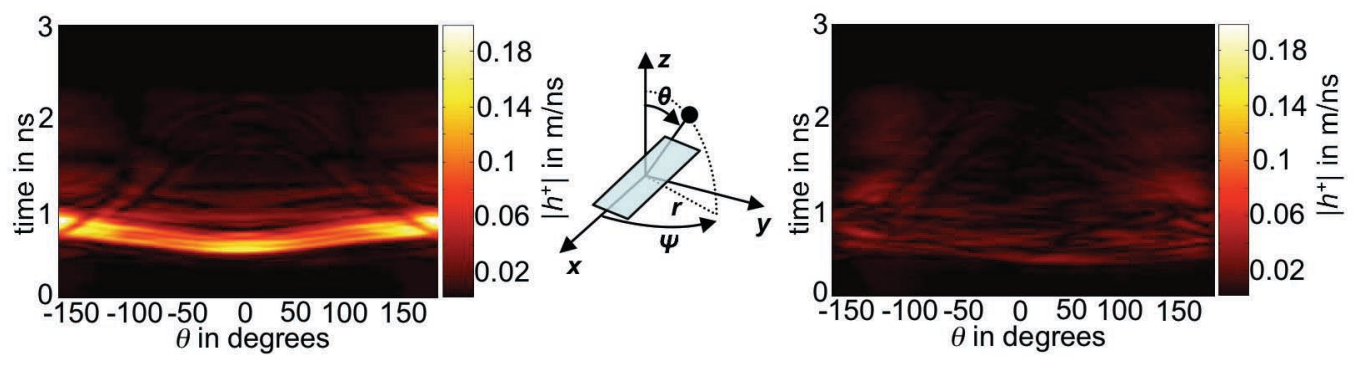

Fig.7: Measured transient response for $\psi=90^{\circ}$, different $\theta$ for the co-polarization (left) and crosspolarization (right) component and the DUT board position with respect to the axes system.

feed a two-element UWB array. The complete structure has been fabricated and tested through a full 3D measurement. The measured results confirm the theoretical approach and validate the realized structure. In particular, a time domain analysis has been conducted. From that analysis, it has been possible to determine the distortion of the network on the propagating impulse.

\section{Acknowledgment:}

We acknowledge the support of the Joint Research Centre of the European Commission, Ispra, Italy, for the 3D measurements.

\section{References:}

[1] M. E. Bialkowski, A. M. Abbosh, "Design of a Compact UWB Out-of-Phase Power Divider", IEEE Micro. Wirel. Comp. Lett., vol. 17, no.4, Apr. 2007.

[2] C.-H. Ho, L. Fan, K. Chang, "New Uniplanar Coplanar Waveguide HybridRing Couplers and Magic-T's", IEEE Trans. Micro. Theory and Tech., vol. 42, no. 12, Dec. 1994.

[3] J. Powell, A. Chandrakasan, "Differential and Single Ended Elliptical Antennas for 3.1-10.6 GHz Ultra Wideband Communication", Proc. IEEE AP Soc. Int. Symp. 2004, Monterey, CA, Vol. 3, Jun. 2004.

[4] W.Soergel, W.Wiesbeck, "Influence of the Antennas on the Ultra-Wideband Transmission”, EURASIP Journal on Applied Signal Processing, vol. 3, 2005. 